VOL. 9 (2005), 25-41

\title{
On $m$-Quasi-Irresolute Functions
}

\author{
TAKashi NoIri AND VAleriu Popa
}

\begin{abstract}
In this paper we introduce a new notion of $m$-quasi irresolute functions as functions from a set satisfying some minimal conditions into a topological space. We obtain some characterizations and several properties of such functions. This function lead us to the formulation of a unified theory of $(\theta, s)$-continuity [26], $\alpha$-quasi irresolute [24], weakly $\theta$-irresolute [19], $\theta$-irresolute [27], $\beta$-quasi irresolute [23].
\end{abstract}

\section{INTRODUCTION}

Semi-open sets, preopen sets, $\alpha$-sets, and $\beta$-open-sets play an important role in the research of generalization of continuity in topological spaces. By using these sets several authors introduced and studied various types of modifications of iiresolute functions due to Crossley and Hildebrand [12]. In 1998, Ganster et al. [19] introduced weak $\theta$-irresoluteness. On the other handm Joseph and Kwack [26] introduced the concept of $(\theta, s)$-continuous functions. Quite recently, Jafari and Noiri [24] introduced and investigated the notion of $\alpha$-quasi-irresoluteness which is between $(\theta, s)$-continuity and weak $\theta$-irresoluteness. In [23], Jafari and Noiri introduced and studied the notion of $\beta$-quasi-irresoluteness which is weaker than one of weak $\theta$-irresoluteness.

In this paper, in order to unify several properties of the modifications of weak $\theta$ irresoluteness stated above, we introduce a new function, called $m$-quasi-irresolute functions, which is a function from a set satisfying some minimal conditions into a topological space. We obtain several characterizations and properties of such funtions. This functions leads us to the formulation of a unified theory of $(\theta, s)$ continuity, $\alpha$-quasi-irresolute, weakly $\theta$-irresolute, $\theta$-irresolute, $\beta$-quasi-irresolute.

\section{Preliminaries}

Throughout the present paper, $(X, \tau)$ and $(Y, \sigma)$ denote topological spaces. Let $A$ a subset of $X$. The closure of $A$ and the interior of $A$ are denoted by $\mathrm{Cl}(A)$ and $\operatorname{Int}(a)$, respectively. The $\theta$-closure (resp. $\delta$-closure) of $A, \mathrm{Cl}_{\theta}(A)\left(\operatorname{resp} . \mathrm{Cl}_{\delta}(A)\right)$, is defined by the set of all $x \in X$ such that $A \cap \mathrm{Cl}(U) \neq \emptyset($ resp. $A \cap \operatorname{Int}(\mathrm{Cl}(U)) \neq \emptyset)$

2000 Mathematics Subject Classification. 54C08.

Key words and phrases. $m$-structure, $(\theta, s)$-continuous, $\alpha$-quasi-irresolute, weakly $\theta$-irresolute, $\beta$-quasi-irresolute, $m$-compact, $S$-closed, $m$-quasi-closed graph. 
for any open set $U$ containing $x$. A subset $A$ is said to be $\theta$-closed (resp. $\delta$-closed) [50] if $A=\mathrm{Cl}_{\theta}(A)$ (resp. $A=\mathrm{Cl}_{\delta}(A)$ ). The complement of a $\theta$-closed (resp. $\delta$-closed) set is said to be $\theta$-open (resp. $\delta$-open). A subset $A$ is said to be regular closed (resp. regular open if $\mathrm{Cl}(\operatorname{Int}(A))=A$ (resp. Int $(\mathrm{Cl}(A))=A$ ). The set $\{x \in X: x \in U \subset A$ for some $U \in \operatorname{RO}(X, \tau)\}$ is said to be the $\delta$-interior of $A$ and is denoted by $\operatorname{Int}_{\delta}(A)$, where $\operatorname{RO}(X, \tau)$ is the family of regular open sets of $(X, \tau)$.

Definition 2.1. Let $(X, \tau)$ be a topological space. A subset $A$ of $X$ is said to be

(1) semi-open [28] if $A \subset \mathrm{Cl}(\operatorname{Int}(A))$,

(2) preopen [32] if $A \subset \operatorname{Int}(\mathrm{Cl}(A))$,

(3) $\alpha$-open [37] if $A \subset \operatorname{Int}(\mathrm{Cl}(\operatorname{Int}(A)))$,

(4) $\beta$-open [1] or semi-preopen [4] if $A \subset \mathrm{Cl}(\operatorname{Int}(\mathrm{Cl}(A)))$,

(5) b-open [5] if $A \subset \mathrm{Cl}(\operatorname{Int}(A)) \cup \operatorname{Int}(\mathrm{Cl}(A))$,

(6) $\delta$-semi-open [42] if $A \subset \mathrm{Cl}\left(\operatorname{Int}_{\delta}(A)\right)$,

(7) $\delta$-preopen [46] if $\left.A \subset \operatorname{IntCl}_{\delta}(A)\right)$.

The family of all semi-open (resp. preopen, $\alpha$-open, semi-preopen, $b$-open, $\delta$-semi-open, $\delta$-preopen, $\theta$-open, $\delta$-open) sets in $X$ is denoted by $\mathrm{SO}(X)$ (resp. $\left.\mathrm{PO}(X), \alpha(X), \beta(X), \operatorname{SPO}(X), \mathrm{BO}(X), \delta \mathrm{SO}(X), \delta \mathrm{PO}(X), \tau_{\theta}, \tau_{\delta}\right)$. The family of all semi-open sets of $X$ containing $x$ is denoted by $\mathrm{SO}(X, x)$.

Definition 2.2. The complement of a semi-open (resp. preopen, $\alpha$-open, $\beta$-open, semi-preopen, $b$-open, $\delta$-semi-open, $\delta$-preopen) set is said to be semi-closed [11] (resp. pre-closed [32], $\alpha$-closed [35], $\beta$-closed [1], semi-preclosed [4], b-closed [5], $\delta$-semi-closed [42], $\delta$-preclosed [46]).

Definition 2.3. The intersection of all semi-closed (resp. preclosed $\alpha$-closed, $\beta$-closed, $b$-closed, $\delta$-semi-closed, $\delta$-preclosed) sets of $X$ containing $A$ is called the semi-closure [11] (resp. preclosure [18], $\alpha$-closure [35], $\beta$-closure [2] or semipreclosure [4], b-closure [5], $\delta$-semi-closure [42], $\delta$-preclosure [46]) of $A$ and is denoted by $\operatorname{sCl}(A)$ (resp. $\operatorname{pCl}(A), \alpha \mathrm{Cl}(A),{ }_{\beta} \mathrm{Cl}(A)$ or $\operatorname{spCl}(A), \mathrm{bCl}(A), \mathrm{sCl}_{\delta}(A)$, $\left.\mathrm{pCl}_{\delta}(A)\right)$.

Definition 2.4. The union of all semi-open(resp. preopen, $\alpha$-open, $\beta$-open, $b$ open, $\delta$-semi-open, $\delta$-preopen) sets of $X$ contained in $A$ is called the semi-interior (resp. preinterior, $\alpha$-interior, $\beta$-interior or semi-preinterior, $b$-interior, $\delta$-semiinterior, $\delta$-preinterior) of $A$ and is denoted by $\operatorname{sint}(A)(\operatorname{resp} . \operatorname{pInt}(A), \alpha \operatorname{Int}(A)$, ${ }_{\beta} \operatorname{Int}(A)$ or $\left.\operatorname{spInt}(A), \operatorname{bInt}(A), \operatorname{sInt}_{\delta}(A), \operatorname{pInt}_{\delta}(A)\right)$.

A point $x \in X$ is said to be a $\theta$-semi-cluster point of a subset $S$ of $X$ if $S \cap \mathrm{Cl}(U) \neq \emptyset$ for every $U \in \mathrm{SO}(X, x)$. The set of all $\theta$-semi-cluster points of $S$ is called the $\theta$-semi-closure of $S$ and is denoted by $\theta-\mathrm{sCl}(S)$. A subset $S$ is said to be $\theta$-semi-closed [26] if $S=\theta-\mathrm{sCl}(S)$. The complement of a $\theta$-semi-closed set is said to be $\theta$-semi-open. Tha family of all $\theta$-semi-open sets of $X$ is denoted by $\theta \mathrm{SO}(X)$. 
Definition 2.5. A function $f:(X, \tau) \rightarrow(Y, \sigma)$ is said to be $s$-continuous [9] or strongly semi-continuous [3] (resp. strongly $\alpha$-continuous [7], irresolute [12], semi-preirresolute [21], almost-irresolute [8]) if for each $x \in X$ and each $V \in$ $\mathrm{SO}(Y, f(x))$, there exists an open (resp. $\alpha$-open, semi-open, preopen, $\beta$-open) set $U$ of $X$ containing $x$ such that $f(U) \subset V$.

Definition 2.6. A function $f:(X, \tau) \rightarrow(Y, \sigma)$ is said to be $(\theta, s)$-continuous [26] (resp. ( $p, s)$-continuous [22], $\theta$-irresolute [27], weakly-irresolute [19], $\alpha$-quasiirresolute [24], $\beta$-quasi-irresolute [23]) if for each $x \in X$ and each $V \in \operatorname{SO}(Y, f(x))$, there exists an open (resp. preopen, $\theta$-semi-open, semi-open, $\alpha$-open, $\beta$-open) set $U$ of $X$ containing $x$ such that $f(U) \subset \mathrm{Cl}(V)$.

\section{3. $m$-QUASI-IRRESOLUTE FUNCTIONS}

Definition 3.1. A subfamily $m_{X}$ of the power set $\mathcal{P}(X)$ of a nonempty set $X$ is called a minimal structure (briefly $m$-structure) on $X$ if $m$ satisfies the following properties: $\emptyset \in m_{X}$ and $X \in m_{X}$.

By $\left(X, m_{X}\right)$, we denote a nonempty subset $X$ with normal structure $m_{X}$ on $X$. We call the pair $\left(X, m_{X}\right)$ an $m$-space. Each member of $m_{X}$ is said to be $m_{X^{-}}$open (briefly $m$-open) and the complement of an $m_{X}$-open set is said to be $m_{X}$-closed (briefly $m$-closed).

Definition 3.2. A minimal strcture $m_{X}$ on a nonempty set $X$ is said to have property $(\mathcal{B})[31]$ if the union of any family of subsets belonging to $m_{X}$ belongs to $m_{X}$.

Remark 3.1. An $m$-structure with the property $(\mathcal{B})$ is called a generalized topology by Luhojan [29]. Császár [13] called a family $m$ a generalized topology if it satisfies $\emptyset \in m$ and has the property $(\mathcal{B})$. Mashhour et al. [34] called a family $m$ supra-topology if it satisfies $X \in m$ and has the property $(\mathcal{B})$. In the present paper, we do not always assume the property $(\mathcal{B})$ on $m$-strctures.

Remark 3.2. Let $(X, \tau)$ be a topological space. Then the families $\tau, \operatorname{SO}(X)$, $\mathrm{PO}(X), \alpha(X), \beta(X), \delta \mathrm{PO}(X), \delta \mathrm{SO}(X), \mathrm{BO}(X), \tau_{\theta}$ and $\tau_{\delta}$ are all $m$-structures on $X$ with the property $(\mathcal{B})$. It is well-known that $\tau_{\theta}, \tau_{\delta}$ and $\alpha(X)$ are topologies for $X$ and the others are not topologies.

Definition 3.3. Let $X$ be a nonempty set and $m_{X}$ an $m$-structure on $X$. For a subset $A$ of $X$, the $m_{X}$-closure of $A$ and the $m_{X}$-interior of $A$ are defined in [31] as follows:

(1) $\operatorname{mCl}(A)=\cap\left\{F: A \subset F, X-F \in m_{X}\right\}$,

(2) $\operatorname{mInt}(A)=\cup\left\{U: U \subset A, U \in m_{X}\right\}$.

Remark 3.3. Let $(X, \tau)$ be a topological space and $A$ a subset of $X$. If $m_{X}=\tau$ (resp. $\mathrm{SO}(X), \mathrm{PO}(X), \alpha(X), \beta(X), \delta \mathrm{PO}(X), \delta \mathrm{SO}(X), \mathrm{BO}(X))$, then we have

(1) $\operatorname{mCl}(A)=\mathrm{Cl}(A)\left(\right.$ resp. $\mathrm{sCl}(A), \operatorname{pCl}(A), \alpha \mathrm{Cl}(A),{ }_{\beta} \mathrm{Cl}(A), \mathrm{pCl}_{\delta}(A), \mathrm{sCl}_{\delta}(A)$, $\mathrm{bCl}(A))$, 
(2) $\operatorname{mInt}(A)=\operatorname{Int}(A)\left(\operatorname{resp} . \operatorname{sInt}(A), \operatorname{pInt}(A), \alpha \operatorname{Int}(A),{ }_{\beta} \operatorname{Int}(A), \operatorname{pInt}_{\delta}(A)\right.$, $\left.\operatorname{sInt}_{\delta}(A), \operatorname{bInt}(A)\right)$.

Lemma 3.1 (Maki [31]). Let $X$ be a nonempty set and $m_{X}$ a minimal strcture on $X$. For subsets $A$ and $B$ of $X$, the following properties hold:

(1) $m C l(X-A)=X-m \operatorname{Int}(A))$ and $m \operatorname{Int}(X-A)=X-m C l(A))$,

(2) If $(X-A) \in m_{X}$, then $m C l(A)=A$ and if $A \in m_{X}$, then $m \operatorname{Int}(A)=A$,

(3) $m C l(\emptyset)=\emptyset, m C l(X)=X, \operatorname{mInt}(\emptyset)=\emptyset$ and $m \operatorname{Int}(X)=X$,

(4) If $A \subset B$, then $m C l(A) \subset m C l(B)$ and $\operatorname{mInt}(A) \subset m \operatorname{Int}(B)$,

(5) $A \subset m C l(A)$ and $m \operatorname{Int}(A) \subset A$,

(6) $m C l(m C l(A))=m C l(A)$ and $m \operatorname{Int}(m \operatorname{Int}(A))=m \operatorname{Int}(A)$.

Lemma 3.2 (Popa and Noiri [43]). Let $X$ be a nonempty set with a minimal structure $m_{X}$ and $A$ a subset of $X$. Then $x \in m C l(A)$ if and only if $U \cap A \neq \emptyset$ for every $U \in m_{X}$ containing $x$.

Lemma 3.3 (Popa and Noiri [43]). Let $X$ be a nonempty set and $m_{X}$ a minimal structure on $X$ satisfying the property $(\mathcal{B})$. For a subset $A$ of $X$, the following properties hold:

(1) $A \in m_{X}$ if and only if $m \operatorname{Int}(A)=A$,

(2) $A$ is $m_{X}$-closed if and only if $m C l(A)=A$,

(3) $\operatorname{mInt}(A) \in m_{X}$ and $m C l(A)$ is $m_{X}$-closed.

Definition 3.4. A function $f:\left(X, m_{X}\right) \rightarrow(Y, \sigma)$ is said to be $m$-irresolute (resp. $m$-quasi-irresolute) at $x \in X$ if for each semi-open set $V$ of $(Y, \sigma)$ containing $f(x)$, there exists $U \in m_{X}$ containing $x$ such that $f(U) \subset V$ (resp. $f(U) \subset \mathrm{Cl}(V)$ ). A function $f:\left(X, m_{X}\right) \rightarrow(Y, \sigma)$ is said to be $m$-irresolute (resp. $m$-quasi-irresolute) if it has the property at each point $x \in X$.

Remark 3.4. Let $f:(X, \tau) \rightarrow(Y, \sigma)$ be a function.

(1) If $m_{x}=\tau$ (resp. $\left.\mathrm{SO}(X), \mathrm{PO}(X), \alpha(X), \beta(X)\right)$ and $f:\left(X, m_{X}\right) \rightarrow(Y, \sigma)$ is $m$-irresolute, then $f$ is $s$-continuous or strongly semi-continuous (resp. irresolute, semi-preirresolute, strongly $\alpha$-continous, almost-irresolute).

(2) If $m_{X}=\tau$ (resp. $\left.\mathrm{SO}(X), \theta \mathrm{SO}(X), \mathrm{PO}(X), \alpha(X), \beta(X)\right)$ and $f:\left(X, m_{X}\right) \rightarrow$ $(Y, \sigma)$ is $m$-quasi-irresolute, then $f$ is $(\theta, s)$-continuous (resp. weakly $\theta$ irresolute, $\theta$-irresolute, $(p, s)$-continuous, $\alpha$-quasi-irresolute, $\beta$-quasi-irresolute).

Theorem 3.1 (Popa and Noiri [43]). For a function $f:\left(X, m_{X}\right) \rightarrow(Y, \sigma)$, the following properties are equivalent:

(1) $f$ is m-irresolute;

(2) $f^{-1}(V)=m \operatorname{mint}\left(f^{-1}(V)\right)$ for every $V \in S O(Y)$;

(3) $f(m C l(A)) \subset s C l(f(A))$ for every subset $A$ of $X$;

(4) $m C l\left(f^{-1}(B)\right) \subset f^{-1}(s C l(B))$ for every subset $B$ of $Y$;

(5) $f^{-1}(\operatorname{sInt}(B)) \subset m \operatorname{mint}\left(f^{-1}(B)\right)$ for every subset $B$ of $Y$;

(6) $m C l\left(f^{-1}(K)\right)=f^{-1}(K)$ for every semi-closed set $K$ of $Y$. 
Corollary 3.1. For a function $f:\left(X, m_{X}\right) \rightarrow(Y \sigma)$, where $m$ satisfies the property $(\mathcal{B})$, the following properties are equivalent:

(1) $f$ is m-irresolute;

(2) $f^{-1}(V)$ is $m$-open for every semi-open set $V$ of $Y$;

(3) $f^{-1}(K)$ is $m$-closed for every semi-closed set $K$ of $Y$.

Proof. This follows immediately from Theorem 3.1 and Lemma 3.3.

Remark 3.5. Let $f:(X, \tau) \rightarrow(Y, \sigma)$ be a function. We put $m=\tau(\operatorname{resp} . \operatorname{SO}(X)$, $\mathrm{PO}(X), \alpha(X), \beta(X))$. Then by Theorem 3.1 we obtain the characterizations established in [3] and [9] (resp. [12], [21], [8]).

Theorem 3.2. A fnction $f:\left(X, m_{X}\right) \rightarrow(Y, \sigma)$ is m-quasi-irresolute at $x \in X$ if and only if for each semi-open set $V$ containing $f(x), \quad x \quad \in$ $\operatorname{mInt}\left(f^{-1}(C l(V))\right)$.

Proof. Necessity. Let $f$ be $m$-quasi-irresolute at $x$ and $V$ a semi-open set containing $f(x)$. Then $\mathrm{m}$ there exists $U \in m_{X}$ containing $x$ such that $f(U) \subset \mathrm{Cl}(V)$. Then we have $x \in U \subset f^{-1}(\mathrm{Cl}(V))$ and hence $x \in \operatorname{mInt}\left(f^{-1}(\mathrm{Cl}(V))\right)$.

Sufficiency. Let $V$ be a semi-open set containing $f(x)$. Then, by the hypothesis we have $x \in \operatorname{mInt}\left(f^{-1}(\mathrm{Cl}(V))\right)$. There exists $U \in m_{X}$ such that $x \in U$ and $U \subset f^{-1}(\mathrm{Cl}(V))$; hence $f(U) \subset \mathrm{Cl}(V)$. This shows that $f$ is $m$-quasi-irresolute at $x \in X$.

Theorem 3.3. For a function $f:\left(X, m_{X}\right) \rightarrow(Y, \sigma)$, the following properties are equivalent:

(1) $f$ is m-quasi-irresolute;

(2) $f^{-1}(V) \subset \operatorname{mInt}\left(f^{-1}(C l(V))\right)$ for every semi-open set $V$ of $Y$;

(3) $m C l\left(f^{-1}(\operatorname{Int}(F))\right) \subset f^{-1}(F)$ for every semi-closed set $F$ of $Y$;

(4) $m C l\left(f^{-1}(\operatorname{Int}(s C l(B))) \subset f^{-1}(s C l(B))\right.$ for every subset $B$ of $Y$;

(5) $f^{-1}(\operatorname{sint}(B)) \subset m \operatorname{Int}\left(f^{-1}(C l(\operatorname{sint}(B)))\right)$ for every subset $B$ of $Y$.

Proof.

$(1) \Rightarrow(2)$ : Let $V$ be any semi-open set of $Y$ and $x \in f^{-1}(V)$. Then $f(x) \in V$. Since $f$ is $m$-quasi-irresolute at $x$, by Theorem 3.2 we have $x \in \operatorname{mInt}\left(f^{-1}(\mathrm{Cl}(V))\right)$ and hence $f^{-1}(V) \subset \operatorname{mInt}\left(f^{-1}(\mathrm{Cl}(V))\right)$.

$(2) \Rightarrow(3)$ : Let $F$ be any semi-closed set of $Y$. Then $Y-F$ is semi-open in $Y$ and by (2) and Lemma 3.1 we have

$$
\begin{gathered}
X-f^{-1}(F)=f^{-1}(Y-F) \subset \operatorname{mInt}\left(f^{-1}(\mathrm{Cl}(Y-F))\right) \\
=\operatorname{mInt}\left(f^{-1}(Y-\operatorname{Int}(F))\right)=\operatorname{mInt}\left(X-f^{-1}(\operatorname{Int}(F))\right)=X-\operatorname{mCl}\left(f^{-1}(\operatorname{Int}(F))\right) .
\end{gathered}
$$

Therefore, we have $\mathrm{mCl}\left(f^{-1}(\operatorname{Int}(F))\right) \subset f^{-1}(F)$.

$(3) \Rightarrow(4)$ : Let $B$ be any subset of $Y$. Then $\mathrm{sCl}(B)$ is semi-closed in $Y$ and by (3) we obtain $\mathrm{mCl}\left(f^{-1}(\operatorname{Int}(\mathrm{sCl}(B)))\right) \subset f^{-1}(\mathrm{sCl}(B))$.

$(4) \Rightarrow(5)$ : Let $B$ be any subset of $Y$. Then we have

$$
f^{-1}(\operatorname{sint}(B))=X-f^{-1}(\operatorname{sCl}(Y-B))
$$




$$
\subset X-\operatorname{mCl}\left(f^{-1}(\operatorname{Int}(\mathrm{sCl}(Y-B)))\right)=\operatorname{mInt}\left(f^{-1}(\mathrm{Cl}(\operatorname{sint}(B)))\right) \text {. }
$$

$(5) \Rightarrow(1)$ : Let $x \in X$ and $V$ be any semi-open set of $Y$ containing $f(x)$. Then, we have $x \in f^{-1}(V)=f^{-1}(\operatorname{sInt}(V)) \subset \operatorname{mInt}\left(f^{-1}(\mathrm{Cl}(\operatorname{sInt}(V)))\right) \subset \operatorname{mInt}\left(f^{-1}(\mathrm{Cl}(V))\right)$ $\subset \operatorname{mInt}\left(f^{-1}(\mathrm{Cl}(V))\right)$ and hence $x \in \operatorname{mInt}\left(f^{-1}(C l(V))\right)$. By Theorem 3.2, $f$ is $m$-quasi-irresolute at $x$.

Theorem 3.4. For a function $f:\left(X, m_{X}\right) \rightarrow(Y, \sigma)$, the following properties are equaivalent:

(1) $f$ is m-quasi-irresolute;

(2) $f(m C l(A)) \subset \theta-s C l(f(A))$ for every subset $A$ of $X$;

(3) $m C l\left(f^{-1}(B)\right) \subset f^{-1}(\theta-s C l(B))$ for every subset $B$ of $Y$.

Proof.

$(1) \Rightarrow(2)$ : Let $A$ be any subset $X$. Suppose that $x \in \operatorname{mCl}(A)$ and $G$ is any semi-open set of $Y$ containing $f(x)$. Since $f$ is $m$-quasi-irresolute, there exists an $m_{X}$-open set $U$ containing $x$ such that $f(U) \subset \mathrm{Cl}(G)$. Since $x \in \operatorname{mCl}(A)$, by Lemma 3.2 we have $U \cap A \neq \emptyset$. It follows that $\emptyset \neq f(U) \cap f(A) \subset$ $\mathrm{Cl}(G) \cap f(A)$. Hence $\mathrm{Cl}(G) \cap f(A) \neq \emptyset$ and $f(x) \in \theta-\operatorname{sCl}(f(A))$.

$(2) \Rightarrow(3)$ : Let $B$ be any subset of $Y$. Then $f\left(\operatorname{mCl}\left(f^{-1}(B)\right)\right) \subset \theta-$ $\mathrm{sCl}\left(f\left(f^{-1}(B)\right)\right) \subset \theta-\operatorname{sCl}(B)$ and hence $\operatorname{mCl}\left(f^{-1}(B)\right) \subset f^{-1}(\theta-\operatorname{sCl}(B))$.

$(3) \Rightarrow(1)$ : Let $V$ be any semi-open set containing $f(x)$. Since $\mathrm{Cl}(V) \cap(Y-\mathrm{Cl}(V))=\neq$ $\emptyset$, clearly $f(x) \notin \theta-\mathrm{sCl}(Y-\mathrm{Cl}(V))$ and hence $x \notin f^{-1}(\theta-\mathrm{Cl}(Y-\mathrm{Cl}(V)))$. By (3), $x \notin \mathrm{mCl}\left(f^{-1}(Y-\mathrm{Cl}(V))\right)$. By Lemma 3.2, there exists an $m_{X^{-}}$ open set $U$ containing $x$ such that $\left.U \cap f^{-1}(Y-\mathrm{Cl}(V))\right)=\emptyset$; hence $f(U) \cap$ $(Y-\mathrm{Cl}(V))=\emptyset$. This shows that $f(U) \subset \mathrm{Cl}(V)$. Therefore, $f$ is $m$-quasiirresolute.

Theorem 3.5. For a function $f:\left(X, m_{X}\right) \rightarrow(Y, \sigma)$, where $m_{X}$ satisfyies the property $(\mathcal{B})$, the following properties are equivalent:

(1) $f$ is m-quasi-irresolute;

(2) $f^{-1}(F)$ is $m$-open for every regular closed set $F$ of $Y$;

(3) $f^{-1}(V)$ is $m$-closed for every regular open set $V$ of $Y$;

(4) the inverse image of a $\theta$-semi-open set of $Y$ is m-open;

(5) the inverse image of a $\theta$-semi-closed set of $Y$ is $m$-closed.

Proof.

$(1) \Rightarrow(2)$ : Let $F$ be any regular closed set of $Y$. Since $F$ is semi-open, by Theorem 3.3 $f^{-1}(F) \subset \operatorname{mInt}\left(f^{-1}(\mathrm{Cl}(F))\right)=\operatorname{mInt}\left(f^{-1}(F)\right)$. By Lemma 3.1, $f^{-1}(F)=$ $\operatorname{mInt}\left(f^{-1}(F)\right)$ and by Lemma $3.3 f^{-1}(F)$ is $m$-open.

$(2) \Rightarrow(3)$ : This is obvious.

$(3) \Rightarrow(4)$ : This follows from the fact that (i) every $\theta$-emi-open set is the union of regular closed sets and (ii) $m$ satisfies the property $(\mathcal{B})$.

$(4) \Rightarrow(5)$ : This is obvious. 
$(5) \Rightarrow(1)$ : Let $x \in X$ and $V$ be any semi-open set of $Y$ containing $f(x)$. Since $\mathrm{Cl}(V)$ is regular closed, it is $\theta$-semi-open. Now, put $U=f^{-1}(\mathrm{Cl}(V))$, then by (4) $U$ is an $m$-open set containing $x$ and $f(U) \subset \mathrm{Cl}(V)$. This shows that $f$ is $m$-quasi-irresolute.

Remark 3.6. Let $f:(X, \tau) \rightarrow(Y, \sigma)$ be a function. Let $m_{X}=\tau(\operatorname{resp} . \operatorname{SO}(X)$, $\theta \mathrm{SO}(X), \alpha(X), \beta(X))$ and $f:\left(X, m_{X}\right) \rightarrow(Y, \sigma)$ be $m$-quasi-irresolute, then by Theorems 3.4 and 3.5 we obtain the results established in Theorem 7 of [26] (resp. Theorem 1.2 of [19]; Theorem 2.2 of [27], Theorem 2.1 of [24]; Theorem 3.1 of [23]).

Definition 3.5. A function $f:\left(X, m_{X}\right) \rightarrow(Y, \sigma)$ is said to be

(1) contra-m-continuous [41] if $f^{-1}(V)=\operatorname{mCl}\left(f^{-1}(V)\right)$ for every $V \in \sigma$;

(2) almost contra-m-continuous if $f^{-1}(V)=\mathrm{mCl}\left(f^{-1}(V)\right)$ for every reagular open set of $Y$.

Lemma 3.4 (Noiri and Popa [41]). Let $\left(X, m_{X}\right)$ be an m-space, where $m_{X}$ has the property $(\mathcal{B})$. For a function $f:\left(X, m_{X}\right) \rightarrow(Y, \sigma)$, the following property are equaivalent:

(1) $f$ is contra-m-continuous;

(2) $f^{-1}(F)$ is m-open for every closed set $F$ of $Y$;

(3) $f^{-1}(V)$ is $m$-closed for every open set $V$ of $Y$.

Let $(Y, \sigma)$ be a topological space. The family of regular open sets of $Y$ forms a base for a semi-regular topology on $Y$ which is called the semi-regularization of $\sigma$ and is denoted by $\sigma_{s}$.

Theorem 3.6. For a function $f:\left(X, m_{X}\right) \rightarrow(Y, \sigma)$, where $m_{X}$ has the property $(\mathcal{B})$, the assertion (1) implies both (2) and (3) which are equivalent:

(1) $f:\left(X, m_{X}\right) \rightarrow\left(Y, \sigma_{s}\right)$ is contra-m-continuous,

(2) $f:\left(X, m_{X}\right) \rightarrow(Y, \sigma)$ is m-quasi-irresolute,

(3) $f:\left(X, m_{X}\right) \rightarrow(Y, \sigma)$ is almost contra-m-continuous.

Proof. The proof follows from Theorem 3.5 and Lemma 3.4.

\section{Comparasions of fuctions}

Definition 4.1. A function $f:\left(X, m_{X}\right) \rightarrow(Y, \sigma)$ is said to satisfy the $s$ interiority condition if $\operatorname{mInt}\left(f^{-1}(\mathrm{Cl}(V))\right) \subset f^{-1}(V)$ for each semi-open set $V$ of $Y$.

Theorem 4.1. If a function $f:\left(X, m_{X}\right) \rightarrow(T, \sigma)$ is m-quasi-irresolute and satisfies the s-interiority condition, then $f$ is $m$-irresolute.

Proof. Let $V$ be any semi-open set of $Y$. Since $f$ is $m$-quasi-irresolute, by Theorem $3.3 f^{-1}(V) \subset \operatorname{mInt}\left(f^{-1}(\mathrm{Cl}(V))\right)$. By the $s$-interiority condition of $f$ and Lemma 3.1 ,

$$
f^{-1}(V) \subset \operatorname{mInt}\left(f^{-1}(\mathrm{Cl}(V))\right)=\operatorname{mInt}\left(\operatorname{mInt}\left(f^{-1}(\mathrm{Cl}(V))\right)\right.
$$




$$
\subset \operatorname{mInt}\left(f^{-1}(V)\right) \subset f^{-1}(V) .
$$

Therefore, we obtain $f^{-1}(V)=\operatorname{mInt}\left(f^{-1}(V)\right)$. By Theorem 3.1, $f$ is $m$-irresolute.

Definition 4.2. A topological space $(Y, \sigma)$ is said to be strongly semi-regular [25] if for each semi-closed set $K$ and each $y \in Y-K$, there exists a regular closed sets $F$ containing $y$ such that $F \cap K=\emptyset$.

Lemma 4.1 (Jafari and Noiri [25]). For a topological space $(Y, \sigma)$, the following properties are equivalent:

(1) $(Y, \sigma)$ is strongly semi-regular;

(2) for each semi-open set $W$ of $(Y, \sigma)$ and each $y \in W$, there exists a semiopen sets $V$ such that $y \in V \subset C l(V) \subset W$;

(3) for each semi-open set $W$ and each $y \in W$, there exists a regular closed sets $F$ such that $y \in F \subset W$;

(4) $s C l(B)=\theta-s C l(B)$ for every subset $B$ of $Y$,

(5) every semi-open set of $(Y, \sigma)$ is $\theta$-semi-open.

Theorem 4.2. Let $(Y, \sigma)$ be strongly semi-regular. Then, for a function $f$ : $\left(X, m_{X}\right) \rightarrow(Y, \sigma)$ the following properties are equivalent:

(1) $f$ is m-irresolute;

(2) $f^{-1}(\theta-s C l(B))=m C l\left(f^{-1}(\theta-s C l(B))\right)$ for every subset $B$ of $Y$;

(3) $f$ is m-quasi-irresolute;

(4) $f^{-1}(F)=m C l\left(f^{-1}(F)\right)$ for every $\theta$-semi-closed set $F$ of $Y$;

(5) $f^{-1}(V)=m \operatorname{Int}\left(f^{-1}(V)\right)$ for every $\theta$-semi-open set $V$ of $Y$.

Proof.

$(1) \Rightarrow(2)$ : Let $B$ be any subset of $Y$. By Lemma $4.1, \theta-\operatorname{sCl}(B)$ is semi-closed and by Theorem 3.1, $f^{-1}(\theta-\operatorname{sCl}(B))=\operatorname{mCl}\left(f^{-1}(\theta-\operatorname{sCl}(B))\right)$.

$(2) \Rightarrow(3)$ : Let $B$ be any subset of $Y$. Then, by (2) and Lemma 3.1, we have $\operatorname{mCl}\left(f^{-1}(B)\right) \subset \operatorname{mCl}\left(f^{-1}(\theta-\operatorname{sCl}(B))\right)=f^{-1}(\theta-\operatorname{sCl}(B))$. Therefore, by Theorem $3.4 f$ is $m$-quasi-irresolute.

$(3) \Rightarrow(4)$ : Let $F$ be any $\theta$-semi-closed set of $Y$. By Theorem 3.4 and Lemma 3.1, we have $\mathrm{mCl}\left(f^{-1}(F)\right) \subset f^{-1}(\theta-\operatorname{sCl}(F))=f^{-1}(F)$. Therefore, we obtain $f^{-1}(F)=\operatorname{mCl}\left(f^{-1}(F)\right)$ for every $\theta$-semi-closed set of $Y$.

$(4) \Rightarrow(5)$ : Let $V$ be any $\theta$-semi-open set of $Y$. Then $Y-V$ is $\theta$-semi-closed set of $Y$ and by (4) and Lemma 3.1, we have $X-\operatorname{mInt}\left(f^{-1}(V)\right)=\operatorname{mCl}\left(f^{-1}(Y-\right.$ $V))=f^{-1}(Y-V)=X-f^{-1}(V)$. Therefore, we obtain $f^{-1}(V)=$ $\operatorname{mInt}\left(f^{-1}(V)\right)$.

$(5) \Rightarrow(1)$ : Let $V$ be any open semi-open set of $Y$. Since $(Y, \sigma)$ is strongly semiregular, by Lemma $4.1 V$ is $\theta$-semi-open. By (5) $f^{-1}(V)=\operatorname{mInt}\left(f^{-1}(V)\right)$ and hence by Theorem $3.1 f$ is $m$-irresolute. 
Remark 4.1. By Theorem 4.2, we obtain the further characterization of $(\theta, s)$ continuity, $(p, s)$-continuity, weak $\theta$-irresoluteness, $\theta$-irresoluteness, $\alpha$-quasi-irresoluteness and $\beta$-quasi-irresoluteness.

We recall that a topological space $(Y \sigma)$ is called extremally disconnected if the closure of each open set of $Y$ is open if $Y$.

Definition 4.3. A function $f:\left(X, m_{X}\right) \rightarrow(Y, \sigma)$ is said to be weakly $m$ continuous [44] (resp. almost $m$-continuous [45]) if for each $x \in X$ and each open set $V$ containing $f(x)$, there exists $U \in m_{X}$ containing $x$ such that $f(U) \subset \mathrm{Cl}(V)$ (resp. $f(U) \subset \operatorname{Int}(\mathrm{Cl}(V)))$.

Theorem 4.3. Let $\left(X, m_{X}\right)$ be an $m$-space, where $m_{X}$ has the property $(\mathcal{B})$, and $(Y, \sigma)$ an extremally disconnected space. For a function $f:\left(X, m_{X}\right) \rightarrow(Y, \sigma)$, the following properties are equaivalent:

(1) $f$ is m-quasi-irresolute;

(2) $f$ is almost m-continuous;

(3) $f$ is weakly m-continuous.

Proof.

$(1) \Rightarrow(2)$ : Let $G$ be any open set of $Y$ containing $f(x)$. There exists an $m$-open set $U$ containing $x$ such that $f(U) \subset \mathrm{Cl}(V)$. Since $(Y, \sigma)$ is extremally disconnected, $\mathrm{Cl}(V)$ is open in $(Y, \sigma)$ and $\mathrm{Cl}(V)=\operatorname{Int}(\mathrm{Cl}(V))$. Hence $f$ is almost $m$-continuous.

$(2) \Rightarrow(3)$ : This is obvious.

$(3) \Rightarrow(1)$ : Let $F$ be any regular closed set of $(Y, \sigma)$. Since $(Y, \sigma)$ is extremally disconnected, $F$ is open and since $f$ is weakly $m$-continous, by Theorem 3.1 of [44] $f^{-1}(F) \subset \operatorname{mInt}\left(f^{-1}(\mathrm{Cl}(F))\right)=\operatorname{mInt}\left(f^{-1}(F)\right)$. Therefore, we obtain $f^{-1}(F)=\operatorname{mInt}\left(f^{-1}(F)\right)$. Since $m_{X}$ has the property $(\mathcal{B})$, by Lemma 3.3 $f^{-1}(F)$ is $m$-open and hence by Theorem $3.5 f$ is $m$-quasi-ireesolute.

Remark 4.2. By Theorem 4.3, we obtain the further characterization of $(\theta, s)$ continuity, $(p, s)$-continuityy, weak $\theta$-irresoluteness, $\theta$-irresoluteness, $\alpha$-quasi-irresoluteness and $\beta$-quasi-irresoluteness.

\section{Some PROPERTIES OF $m$-QUASI-IRRESOLUTE FUnCTIONS}

Definition 5.1. An $m$-space $\left(X, m_{X}\right)$ is said to be $m$-Hausdorff [43] if for each pair of distinct points $x, y \in X$, there exists $U, V \in m_{X}$ containing $x$ and $y$, respectively, such that $U \cap V=\emptyset$.

Definition 5.2. A topological space $(Y, \sigma)$ is said to be

(1) weakly Hausdorff [47] if each element of $Y$ is the intersection of regular closed sets, 
(2) $s$-Urysohn [6] if for each pair of distinct points $x$ and $y$ in $Y$, there exists semi-open sets $U, V$ containing $x$ and $y$, respectively, such that $\mathrm{Cl}(U) \cap$ $\mathrm{Cl}(V)=\emptyset$.

Theorem 5.1. If $f:\left(X, m_{X}\right) \rightarrow(Y, \sigma)$ is an m-quasi-irresolute injection and $(Y, \sigma)$ is s-Urysohn, then $\left(X, m_{X}\right)$ is m-Hausdorff.

Proof. Let $x, y$ be any pair of distinct points of $X$. Then $f(x) \neq f(y)$. Since $(Y, \sigma)$ is $s$-Urysohn, there exists $U, V \in \mathrm{SO}(Y, f(x))$ containing $f(x)$ and $f(y)$, respectively, such that $\mathrm{Cl}(U) \cap \mathrm{Cl}(V)=\emptyset$. Since $f$ is $m$-quasi-irresolute, there exist $g, H \in m_{X}$ containing $x, y$, respectively, such that $f(G) \subset \mathrm{Cl}(U)$ and $f(H) \subset$ $\mathrm{Cl}(V)$. This implies that $G \cap H=\emptyset$. Hence $\left(X, m_{X}\right)$ is $m$-Hausdorff.

Remark 5.1. Let $f:(X, \tau) \rightarrow(Y, \sigma)$ be a function. If $m_{X}=\tau$ (resp. $\mathrm{SO}(X)$, $\theta \mathrm{SO}(X), \alpha(X), \beta(X))$ and $f:\left(X, m_{X}\right) \rightarrow(Y, \sigma)$ is $m$-quasi-irresolute, then by Theorem 5.1 we obtain the results established in Theorem 3.2 of [40] (resp. Proposition 1.7 of [19], Theorem 2.5 of [27], Theorem 3.3 of [24], Theorem 3.2 of [23]).

Definition 5.3. A function $f:\left(X, m_{X}\right) \rightarrow(Y, \sigma)$ is said to have an m-quasi-closed graph if for each $(x, y) \in(X \times Y)-G(f)$, there exist an $m$-open set $U$ containing $x$ and a semi-open set $V$ of $Y$ containing $y$ such that $[U \times \mathrm{Cl}(V)] \cap G(f)=\emptyset$.

Remark 5.2. Let $f:(X, \tau) \rightarrow(Y, \sigma)$ be a function and $m_{X}=\tau$ (resp. $\alpha(X)$, $\beta(X))$. Then the $m$-quasi-closed graph of a function $f:\left(X, m_{X}\right) \rightarrow(Y, \sigma)$ is said to be $(\tau, s)$-closed [40] (resp. $\alpha$-quasi-closed [24], $\beta$-quasi closed [23]).

Lemma 5.1. A function $f:\left(X, m_{X}\right) \rightarrow(Y, \sigma)$ has an $m$-quasi-closed graph if and only if for each $(x, y) \in(X \times Y)-G(f)$, there exist an m-open set $U$ containing $x$ and a semi-open set $V$ of $Y$ containing $y$ such that $f(U) \cap C l(V)=\emptyset$.

Theorem 5.2. If $f:\left(X, m_{X}\right) \rightarrow(Y, \sigma)$ is an m-quasi-irresolute function and $(Y, \sigma)$ is s-Urysohn, then $G(f)$ is m-quasi-closed.

Proof. Suppose that $(x, y) \in(X \times Y)-G(f)$. Then $y \neq f(x)$. Since $Y$ is $s$-Urysohn there exist semi-open sets $V$ and $W$ i $Y$ containing $y$ and $f(x)$, respectively, such that $\mathrm{Cl}(V) \cap \mathrm{Cl}(W)=\emptyset$. Since $f$ is $m$-quasi-irresolute, there exists an $m$-open set $U$ containing $x$ such that $f(U) \subset \mathrm{Cl}(W)$. This implies that $f(U) \cap \mathrm{Cl}(V)=\emptyset$ and by Lemma $5.1 G(f)$ is $m$-quasi-closed.

Remark 5.3. Let $f:(X, \tau) \rightarrow(Y, \sigma)$ be a function and $m_{X}=\tau$ (resp. $\alpha(X)$, $\beta(X), \theta \mathrm{SO}(X))$. If a function $f\left(X, m_{X}\right) \rightarrow(Y, \sigma)$ is $m$-quasi-irresolute, then by Theorem 5.2 we obtain the results established in Theorem 4.1 of [40] (resp. Theorem 4.1 of [24], Theorem 3.1 of [23], Thorem 2.7 of [27]).

Theorem 5.3. If $f:\left(X, m_{X}\right) \rightarrow(Y, \sigma)$ is an injective $m$-quasi-irresolute function with an m-quasi-closed graph, then $\left(X, m_{X}\right)$ is m-Hausdorff.

Proof. Let $x$ and $y$ be any distinct point of $X$. Then, since $f$ is injective, we have $f(x) \neq f(y)$. Then we have $(x, f(y)) \in(X \times Y)-G(f)$. Since $G(f)$ is 
$m$-quasi-closed, by Lemma 5.1 there exist an $m$-open set $U$ of $X$ containing $x$ and a semi-open set $V$ containing $f(y)$ such that $f(U) \cap \mathrm{Cl}(V)=\emptyset$. Since $f$ is $m$-quasi-irresolute, there exists $G \in m_{X}$ containing $y$ such that $f(G) \subset \mathrm{Cl}(V)$. Therefore, we have $f(U) \cap f(G)=\emptyset$. Clearly, we obtain $U \cap G=\emptyset$. This shows that $X$ is $m$-Hausdorff.

Theorem 5.4. If $f:\left(X, m_{X}\right) \rightarrow(Y, \sigma)$ is a surjection and $G(f)$ is m-quasiclosed, then $(Y, \sigma)$ is weakly-Hausdorff.

Proof. Let $y_{1}$ and $y$ be any distinct points of $Y$. Then, since $f$ is surjective, there exists $x \in X$ such that $f(x)=y_{1}$. Then $(x, y) \notin G(f)$ and by Lemma 5.1 there exist $U \in m_{X}$ containing $x$ and $V \in \mathrm{SO}(Y, y)$ such that $f(U) \cap \mathrm{Cl}(V)=\emptyset$. Since $y_{1} \in f(U), y_{1} \notin \mathrm{Cl}(V)$ and $\mathrm{Cl}(V)$ is a regular closed set containing $y$. This shows that $(Y, \sigma)$ is weakly-Hausdorff.

Remark 5.4. Let $f:(X, \tau) \rightarrow(Y, \sigma)$ be a function and $m_{X}=\tau(\operatorname{resp} . \alpha(X)$, $\beta(X))$. If a function $f:\left(X, m_{X}\right) \rightarrow(Y, \sigma)$ is $m$-quasi-irresolute, then by Theorem 5.4 we obtain the results established in Theorem 4.2 of [40] (resp. Theorem 4.2 of [24], Theorem 3.11 of [23]).

Definition 5.4. An $m$-space $\left(X, m_{X}\right)$ is said to be

(1) $m$-connected [43] if $X$ cannot be written as the union of two nonempty disjoint $m$-open sets,

(2) $m$-ultra-connected if $F_{1} \cap F_{2} \neq \emptyset$ for each nonempty $m$-closed sets $F_{1}$ and $\mathrm{F}_{2}$.

We shall recall a topological space $(Y, \sigma)$ is said to be hyperconnected [48] if every nonempty open set is dense.

Theorem 5.5. Let $f:\left(X, m_{X}\right) \rightarrow(Y, \sigma)$ be an m-quasi-irresolute surjection. If $\left(X, m_{X}\right)$ satisfies the property $(\mathcal{B})$ and is $m$-connected, then $(Y, \sigma)$ is connected.

Proof. Assume that $(Y, \sigma)$ is not connected. Then there exist nonempty open sets $V_{1}, V_{2}$ such that $V_{1} \cap V_{2}=\emptyset$ and $V_{1} \cup V_{2}=Y$. Therefore, $V_{1}$ and $V_{2}$ are regular closed. Since $f$ is $m$-quasi-irresolute, by Theorem $3.5 f^{-1}\left(V_{1}\right)$ and $f^{-1}\left(V_{2}\right)$ are $m$-open. Moreover, $X$ is the union of nonempty disjoint sets $f^{-1}\left(V_{1}\right)$ and $f^{-1}\left(V_{2}\right)$. This implies that $\left(X, m_{X}\right)$ is not $m$-connected. This is contrary to the hypothesis that $\left(X, m_{X}\right)$ is $m$-connected. Therefore, $(Y, \sigma)$ is connected.

Theorem 5.6. Let $f:\left(X, m_{X}\right) \rightarrow(Y, \sigma)$ be an $m$-quasi-irresolute surjection. If $\left(X, m_{X}\right)$ satisfies the property $(\mathcal{B})$ and is $m$-ultra-connected, then $(Y, \sigma)$ is hyperconnected.

Proof. Assume that $(Y, \sigma)$ is not hyperconnected. Then there exists an open set $V$ such that $V$ is not dense in $Y$. Now, put $V_{1}=\operatorname{Int}(\mathrm{Cl}(V))$ and $V_{2}=Y-\mathrm{Cl}(V)$. Then $V_{1}$ and $V_{2}$ are nonempty disjoint regular open sets. By Theorem $3.5, f^{-1}\left(V_{1}\right)$ and $f^{-1}\left(V_{2}\right)$ are nonempty disjoint $m$-closed. This is contrary that $\left(X, m_{X}\right)$ is $m$-ultra-connected. 
Remark 5.5. Let $f:(X, \tau) \rightarrow(Y, \sigma)$ be a function. If $m_{X}=\tau$ and $f$ : $\left(X, m_{X}\right) \rightarrow(Y, \sigma)$ is $m$-quasi-irresolute, then by Theorem 5.6 we obtain the result established in Theorem 3.5 of [40].

Definition 5.5. A subset $K$ of a nonempty set $X$ with a minimal structure $m_{X}$ is said to be $m$-compact relative to $\left(X, m_{X}\right)$ if any cover of $K$ by $m$-open sets of $X$ has a finite subcover. If $X$ is $m$-compact relative to $\left(X, m_{X}\right)$, then $\left(X, m_{X}\right)$ is said to be m-compact [43].

Definition 5.6. A subset $K$ of a topological space $(Y, \sigma)$ is said to be $S$-closed relative to $(Y, \sigma)[38]$ if for any cover $\left\{V_{\alpha}: \alpha \in \Delta\right\}$ of $K$ by semi-open sets of $Y$ there exists a finite subset $\Delta_{0}$ of $\Delta$ such that $K \subset \cup_{\alpha \in \Delta_{0}} \mathrm{Cl}\left(V_{\alpha}\right)$. If $Y$ is $S$-closed relative to $(Y, \sigma)$, then $(Y, \sigma)$ is said to be $S$-closed [49].

Remark 5.6. Let $(X, \tau)$ be a topological space and $m_{X}=\tau$ (resp. $\alpha(X), \mathrm{SO}(X)$, $\mathrm{PO}(X)$ ). The definition of " $m$-compact" gives one of compact (resp. $\alpha$-compact [30], s-compact [10], strongly compact [33]).

Theorem 5.7. If $f:\left(X, m_{X}\right) \rightarrow(Y, \sigma)$ is an m-quasi-irresolute function and $K$ is $m$-compact relative to $\left(X, m_{X}\right)$, then $f(K)$ is $S$-closed relative to $(Y, \sigma)$.

Proof. Let $K$ be $m$-compact relative to $\left(X, m_{X}\right)$. Let $\left\{V_{\alpha}: \alpha \in \Delta\right\}$ be any cover of $f(K)$ by semi-open sets of $(Y, \sigma)$. For each $x \in K$, there exists $\alpha(x) \in \Delta$ such that $f(x) \in V_{\alpha(x)}$. Since $f$ is $m$-quasi-irresolute, there exists an $m$-open set $U(x)$ containing $x$ such that $f(U(x)) \subset \mathrm{Cl}\left(V_{\alpha(x)}\right)$. The family $\{U(x): x \in K\}$ is a cover of $K$ by $m$-open sets of $X$. Since $K$ is $m$-compact relative to $\left(X, m_{X}\right)$, there exist a finite number of points, say, $x_{1}, x_{2}, \ldots, x_{n}$ in $K$ such that $K \subset \cup\left\{U\left(x_{k}\right): x_{k} \in\right.$ $K, 1 \leq k \leq n\}$. therefore, we obtain

$$
f(K) \subset \cup\left\{f\left(U\left(x_{k}\right)\right): x_{k} \in K, 1 \leq k \leq n\right\} \subset \cup\left\{\mathrm{Cl}\left(V_{\alpha\left(x_{k}\right)}\right): x_{k} \in K, 1 \leq k \leq n\right\} .
$$

This shows that $f(K)$ is $S$-closed relative to $(Y, \sigma)$.

Corollary 5.1. If $f:\left(X, m_{X}\right) \rightarrow(Y, \sigma)$ is an m-quasi-irresolute surjection and $\left(X, m_{X}\right)$ is m-compact, then $(Y, \sigma)$ is $S$-closed.

Remark 5.7. Let $f:\left(X, m_{X}\right) \rightarrow(Y, \sigma)$ be a function. If $m_{X}=\tau$ (resp. $\alpha(X)$, $\mathrm{SO}(X), \theta \mathrm{SO}(X))$ and $f:\left(X, m_{X}\right) \rightarrow(Y, \sigma)$ is $m$-quasi-irresolute, then by Theorem 5.7 and Corollary 5.1 we obtain the results established in Theorem 5.1 of [40] (resp. Theorem 5.2 of 24, Proposition 1.8 of [19], Theorem 2.2 and Corollary 2.3 of [39]).

Definition 5.7. A topological space $(Y, \sigma)$ is said to be

(1) S-Lindelöf [14] if every cover of $Y$ by regular closed sets has a countable subcover,

(2) countably $S$-cover [17] if every countable cover of $Y$ by regular closed sets has a finite subcover.

Definition 5.8. An $m$-space $\left(X, m_{X}\right)$ is said to be 
(1) $m$-Lindelöf if every cover of $X$ by $m$-open sets has a countable subcover,

(2) countably $m$-closed if every countable cover of $Y$ by $m$-open sets has a finite subcover.

Theorem 5.8. Let $f:\left(X, m_{X}\right) \rightarrow(Y, \sigma)$ be an m-quasi-irresolute surjection, where $m_{X}$ has the property $(\mathcal{B})$. Then the following properties hold:

(1) If $\left(X, m_{X}\right)$ is $m$-Lindelöf, then $(Y, \sigma)$ is S-Lindelöf,

(2) If $\left(X, m_{X}\right)$ is countably m-compact, then $(Y, \sigma)$ is countably $S$-closed.

Proof. We prove only statement (1), the proof of (2) being entirely analogous. Let $\left\{V_{\alpha}: \alpha \in \Delta\right\}$ be any cover of $Y$ by regular closed sets of $Y$. Since $f$ is $m$-quasi-irresolute, by Theorem $3.5\left\{f^{-1}\left(V_{\alpha}\right): \alpha \in \Delta\right\}$ is an $m$-open cover $X$ and hence there exists countable subset $\Delta_{0}$ of $\Delta$ such that $X=\cup\left\{f^{-1}\left(V_{\alpha}\right): \alpha \in \Delta_{0}\right\}$. Therefore, we have $Y=\cup\left\{V_{\alpha} \in \Delta_{0}\right\}$. Therefore, $(Y, \sigma)$ is $S$-Lindelöf.

Remark 5.8. Let $f:(X, \tau) \rightarrow(Y, \sigma)$ be a function and $m_{X}=\tau(\operatorname{resp} . \alpha(X))$. Then by Theorem 5.8 we obtain the result established in Theorem 5.1 of [40] (resp. Theorem 5.2 of [24]).

Definition 5.9. Let $\left(X, m_{X}\right)$ be an $m$-space and $A$ be a subset of $X$. The $m$ frontier of $A$, denoted by $\operatorname{mFr}(A)$, is defined by $\operatorname{mFr}(A)=\operatorname{mCl}(A) \cap \operatorname{mCl}(X-A)=$ $\mathrm{mCl}(A)-\operatorname{mInt}(A)$.

Theorem 5.9. The set of all points $x \in X$ at which a function $f:\left(X, m_{X}\right)$ $\rightarrow(Y, \sigma)$ is not $m$-quasi-irresolute is not identical with the union of the $m$-frontiers of the inverse images of the closure of semi-open sets containing $f(x)$.

Proof. Suppose that $f$ is not $m$-quasi-irresolute at $x \in X$. There exists a semiopen set $V$ of $Y$ containing $f(x)$ such that $f(U)$ is not contained in $\mathrm{Cl}(V)$ for every $m$-open set $U$ containing $x$. Then $U \cap\left(X-f^{-1}(\mathrm{Cl}(V))\right) \neq \emptyset$ for every $m$-open set $U$ containing $x$. By Lemma 3.2, $x \in \operatorname{mCl}\left(X-f^{-1}(\mathrm{Cl}(V))\right)$. On the other hand, we have $x \in f^{-1}(V) \subset \operatorname{mCl}\left(f^{-1}(\mathrm{Cl}(V))\right)$ and hence $x \in \operatorname{mFr}\left(f^{-1}(\mathrm{Cl}(V))\right)$.

Conversely, suppose that $f$ is $m$-quasi-irresolute at $x \in X$ and let $V$ be any semiopen set containing $f(x)$. Then by Theorem 3.2 we have $x \in \operatorname{mInt}\left(f^{-1}(\mathrm{Cl}(V))\right)$. Therefore, $x \notin \operatorname{mFr}\left(f^{-1}(\mathrm{Cl}(V))\right)$ for each semi-open set $V$ of $Y$ containing $f(x)$. This completes the proof.

\section{NEW FORMS OF WEAKLY IRRESOLUTE FUNCTIONS}

Let $A$ be a subset of a topological space $(X, \tau)$. A point $x$ in $X$ is called a semi- $\theta$-cluster point of $A$ if $\operatorname{sCl}(U) \cap A \neq \emptyset$ for every $U \in \mathrm{SO}(X)$ containing $x$. The set of all semi- $\theta$-cluster points of $A$ is called the semi- $\theta$-closure [15] of $A$ and is denoted by $\mathrm{sCl}_{\theta}(A)$. A subset $A$ is said to be semi- $\theta$-closed if $A=\mathrm{sCl}_{\theta}(A)$. The complement of a semi- $\theta$-closed set is said to be semi- $\theta$-open. The family of all semi- $\theta$-open sets of $(X, \tau)$ is denoted by $\mathrm{S} \theta \mathrm{O}(X)$.

Lemma 6.1 (Noiri and Popa [41]). For subsets of a topological space $(X, \tau)$, the following properties hold: 
(1) Every $\theta$-open set is $\theta$-semi-open and every $\theta$-semi-open set is semi- $\theta$-open;

(2) Every semi- $\theta$-set is $\delta$-semi-open and a $\delta$-semi-open set is semi-open.

Proof. (1) is obvious from the definitions and (2) is shown in Lemma 7.1 of [41].

By Diagram 1 of [41] and Lemma 6.1, we have the following diagram in which the converses of implications need not be true as shown by the example stated below.

\section{DIAGRAM I}

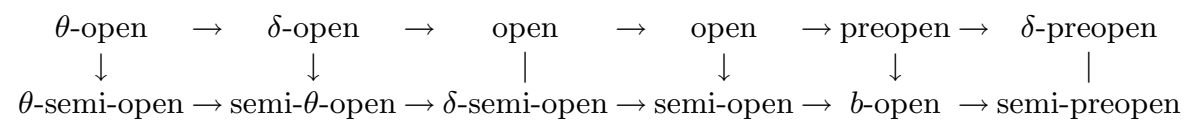

Remark 6.1. In the Diagram above, we have the following properties:

(1) $\delta$-openness and $\theta$-semi-openness are independent of each other as shown by Example 6.1 (below),

(2) It is shown in Example 2 of [42] that openness and $\delta$-semi-openness are independent of each other,

(3) $\delta$-preopenness and semi-preopenness are independent of each other as shown by Examples 6.1 and 6.2 (below).

Example 6.1. Let $X=\{a, b, c\}$ and $\tau=\{X, \emptyset,\{a\},\{b\},\{a, b\}\}$. Then $\{a, b\}$ is a $\delta$-open set of $(X, \tau)$ which is not $\theta$-semi-open. The subset $\{a, c\}$ is a $\theta$-semiopen set which is not $\delta$-preopen and hence not $\delta$-open. Therefore, $\{a, c\}$ is a semi-preopen set which is not $\delta$-preopen.

Example 6.2. Let $X=\{a, b, c, d\}$ and $\tau=\{X, \emptyset,\{a, b\},\{a, b, c\}\}$. Then $\{d\}$ is a $\delta$-preopen set of $(X, \tau)$ which is not semi-preopen.

All families $\tau_{\theta}, \tau_{\delta}, \mathrm{S} \theta \mathrm{O}(X), \mathrm{BO}(X), \delta \mathrm{SO}(X), \delta \mathrm{PO}(X)$ are $m$-structure with the property $(\mathcal{B})$. Especially, $\tau_{\theta}$ and $\tau_{\delta}$ are topologies for $X$. Therefore, we can define $m$-quasi-irresolute function $f:\left(X, m_{X}\right) \rightarrow(Y, \sigma)$, where $m_{X}=\tau_{\theta}, \tau_{\delta}, \mathrm{S} \theta \mathrm{O}(X)$, $\mathrm{BO}(X), \delta \mathrm{SO}(X)$, or $\delta \mathrm{PO}(X)$. Then, we can apply all results obtained in Section $3-5$ to these new functions.

Definition 6.1. A function $f:(X, \tau) \rightarrow(Y, \sigma)$ is said to be $\theta$-quasi-irresolute (resp. $\delta$-quasi-irresolute, $s \theta$-quasi-irresolute, $\delta$ s-quasi-irresolute, $b$-quasi-irresolute, $\delta p$-quasi-irresolute) if for each $V \in \mathrm{SO}(Y, f(x))$, there exists a $\theta$-open (resp. $\delta$ open, semi- $\theta$-open, $\delta$-semi-open, $b$-open, $\delta$-preopen) set $U$ such that $f(U) \subset \mathrm{Cl}(V)$.

By DIAGRAM I, we obtain the following diagram:

\section{DIAGRAM II}

$$
\begin{aligned}
& \theta \text {.q.irr. } \rightarrow \delta \text {.q.irr. } \rightarrow(\theta, s) \text {-c } \rightarrow \alpha \text {-q.irr. } \rightarrow(p, s) \text {-c. } \rightarrow \delta \text { p.q.irr. } \\
& \theta \text {-irr. } \rightarrow \text { s } \theta \text {.q.irr. } \rightarrow \delta \text { s.q.irr. } \rightarrow \text { q. } \theta \text {-irr. } \rightarrow b \text {-q.irr. } \rightarrow \beta \text {-q.irr. }
\end{aligned}
$$


In the diagram above, we abbreviate as follows: c. =continuous, irr. =irresolute.

Lemma 6.2 (Noiri and Popa [41]). Let $(X, \tau)$ be a topological space and $A$ be a subset of $X$.

(1) $A$ is $\delta$-semi-open in $(X, \tau)$ if and only if $A$ is semi-open in $\left(X, \tau_{s}\right)$,

(2) $A$ is $\delta$-preopen in $(X, \tau)$ if and only if $A$ is preopen in $\left(X, \tau_{s}\right)$.

Theorem 6.1. A function $f:(X, \tau) \rightarrow(Y, \sigma)$ is $\delta$-quasi-irresolute (resp. $\delta s$ quasi-irresolute, $\delta p$-quasi-irresolute) if and only if $f:\left(X, \tau_{s}\right) \rightarrow(Y, \sigma)$ is $(\theta, s)$ continuous (resp. quasi- $\theta$-irresolute, $(p, s)$-continuous).

Proof. This is an immediate consequence of Lemma 6.2.

\section{REFERENCES}

[1] M. E. Abd El-Monsef, S. N. El-Deeb and R. A. Mahmoud, $\beta$-open sets and $\beta$ continuous mappings, Bull. Fac. Sci. Assiut Univ. 12(1983), 77-90.

[2] M. E. Abd El-Monsef, R. A. Mahmoud and E. R. Lashin, $\beta$-closure and $\beta$-interior, J. Fac. Ed. Ain Shams Univ 10(1986), 235-245.

[3] M. E. Abd El-Monsef, R. A. Mahmoud and A. A. Nasef, Strongly semi-continuous functions, Arab. J. Phys. Math. Iraq 11(1990).

[4] D. Andrijević, Semi-preopen sets, Mat. Vesnik 38(1986), 24-32.

[5] D. Andrijević, On b-open sets, Mat. Vesnik 48(1996), 53-64.

[6] S. P. Arya and M. P . Bhamini, Some generalizations of pairwise Urysohn spaces, Indian J. Pure Appl. Math. 18(1987), 1088-1093.

[7] Y. Beceren, On strongly $\alpha$-continuous functions, Far East J. Math. Sci. Special Volume (2000), Part I (Geometry and Topology), 51-58.

[8] F. Cammaroto and T. Noiri, Almost irresolute functions, Indian J. Pure Appl. Math. 20(1989), 472-482.

[9] D. E. Cameron and G. Woods, s continuous and s-open mappings (preprint).

[10] D. A. Carnahan, Some Properties Related to Compactness in Topological Spaces, Ph. D. Thesis, Univ. of Arkansas, 1973.

[11] S. G. Crossley i S. K. Hildebrand, Semi-closure, Texas J. Sci. 22(1971), 99-112.

[12] S. G. Crossley i S. K. Hildebrand, Semi-topological properties, Fund. Math. 74(1972), 233254.

[13] Á. Császár, General topology, generalized continuity, Acta Math. Hungar. 96(2002), 351357.

[14] G. Di Maio, S-closed spaces, $S$-sets and S-continuos functions, Accad. Sci. Torino, 118(1984), 125-134.

[15] G. Di Maio and T. Noiri, On s-closed spaces, Indian J. Pure Appl. Math. 18(1987), 226-233.

[16] G. Di Maio and T. Noiri, Weak and strong forms of irresolute functions, Suppl. Rend. Circ. Mat. Palermo (2) 18(1988), 255-273. 
[17] K. Dlaska, N. Ergun and M. Ganster, Countably S-closed spaces, Math. Slovaca 44(1994), 337-348.

[18] S. N. El-Deeb, I. A. Hasanein, A. S. Mashour and T. Noiri, On p-regular spaces, Bull. Math. Soc. Sci. Math. R. S. Roumanie 27(75)(1983), 311-315.

[19] M. Ganster, T. Noiri and I. L. Reilly, Weak and strong forms of $\theta$-irresolute funtions, J. Inst. Math. Comput. Sci. Math. Ser. 1(1988), 19-29.

[20] R. A. Herrmann, rc-convergence, Proc. Amer. Math. Soc. 75(1979), 311-317.

[21] S. Jafari, On semi preirresolute functions, Far East J. Math. Sci. 6(1998), 1003-1010.

[22] S. Jafari, A new type of continuity, (submitted).

[23] S. Jafari and T. Noiri, On $\beta$-quasi-irresolute functions, Mem. Fac. Sci. Kochi Univ. Math. Ser. 21(2000), 53-62.

[24] S. Jafari and T. Noiri, On $\alpha$-quasi-irresolute functions, Rend. Circ. Mat. Palermo (2) 50(2001), 137-152.

[25] S. Jafari and T. Noiri, On strongly semu-regular spaces, Demonstratio Math. 34(2001), 187-190.

[26] J. E. Joseph and M. H. Kwack, On S-closed spaces, Proc. Amer. Math. Soc. 80(1980), 341-348.

[27] F. H. Khedr and T. Noiri, On $\theta$-irresolute functions, Indian J. Math 28(1986), 211-217.

[28] N. Levine, Semi-open sets and semi-continuity in topological spaces, Amer. Math Monthly 70(1963), 36-41.

[29] S. Lugojan, Generalized topology (Romanian), Stud. Cerc. Mat. 34 (1982), 348-360.

[30] S. N. Maheshwar and S. S. Thakur, On $\alpha$-compact spaces, Bull. Inst. Math. Acad. Sinica 13(1985), 341-347.

[31] H. Maki, On generaziling semi-open and preopen sets, Report for Meeting on Topological Spaces Theory and its Applications, August 1996, Yatsushiro College of Technology, pp. $13-18$.

[32] A. S. Mashhour, M. E. Abd El-Monsef and S. N. El-Deep, On precontinuous and weak precontinuous mappings, Proc. Math. Phys. Soc. Egypt 53(1982), 47-53.

[33] A. S. Mashhour, M. E. Abd El-Monsef, I. A. Hasanein and T. Noiri, Strongly compact spaces, Delta J. Sci. 8(1984), 30-46.

[34] A. S. Mashhour, A. A. Allam, F. S. Mahmoud and F. H. Khedr, On supratopological spaces, Indian J. Pure Appl. Math. 14(1983), 502-510.

[35] A. S. Mashhour, I. A. Hasanein and S. N. El-Deep, $\alpha$-continuous and $\alpha$-open mappings, Acta Math. Hungar. 41(1983), 213-218.

[36] M. N. Mukherjee and C. K. Basu, On Semi- $\theta$-closed sets, semi- $\theta$-connectedness and some associated mappings, Bull. Calcutta Math. Soc. 83(1991), 227-238.

[37] O. Njåstad, On some classes of nearly open sets, Pacific J. Math. 15(1965), 961-970.

[38] T. Noiri, On S-closed spaces, Ann. Soc. Sci. Bruxelles 91(1977), 189-194. 
[39] T. Noiri, On S-closed spaces and S-perfect functions, Atti Accad. Sci. Torino 120(1986), $71-79$.

[40] T. Noiri and S. Jafari, Properties of $(\tau, s)$-continuous functions, Topology and its Applications 123(2002), 167-179.

[41] T. Noiri and V. Popa, A unified theory of contra-continuity for functions, Annal. Univ. Sci. Budapesta Math. 44(2002), 115-137.

[42] J. H. Park, B. Y. Lee and M. J. Son, On $\delta$-semiopen sets in topological spaces, J. Indian Acad. Math. 19(1997), 59-67.

[43] V. Popa and T. Noiri, On M-continuous functions, Anal. Univ. "Dunarea de Jos" Galati, Ser. Mat. Fiz. Mec. Teor., Fasc. II, 18(23) (2000), 31-41.

[44] V. Popa and T. Noiri, On weakly m-continuous functions, Mathematica (Cluj) (to appear).

[45] V. Popa and T. Noiri, On almost m-continuous functions (submitted).

[46] S. Raychaudhuri and M. N. Mukherjee, On $\delta$-almost continuity and $\delta$-preopen sets, Bull. Inst. Math. Acad. Sinica 21(1993), 357-366.

[47] T. Soundararajan, Weakly Hausdorff spaces and the cardinality of topological spaces, General Topology and its Relations to Modern Analysis and Algebra III, Proc. Conf. Kapur, 1986, Academia Prague, 1971, pp. 301-306.

[48] L. A. Steen and J. A. Seebach, Counterexamples in Topology, Holt, Rinehart and Winston, New York, 1970.

[49] T. Thompson, S-closed spaces, Proc. Amer. Math. Soc. 60(1976), 335-338.

[50] N. V. Veličko, H-closed topological spaces, Amer. Math. Soc. Transl. (2) 78(1969), 103-118.

\author{
Depatment of Mathematics \\ Yatsushiro College of TeChNology \\ YATSUSHIRO, KUMAMOTO, 866-8501 \\ JAPAN \\ Department of Mathematics \\ UNIVERSITY OF BACǍU \\ 5500 BACǍU \\ RUMUNIA \\ E-mail address: vpopa@ub.ro
}

E-mail address: noiri@as.yatsushiro-nct.ac.jp 